\title{
HPV Vaccine and College-Age Men: A Scoping Review
}

American Journal of Men's Health November-December I-12 (C) The Author(s) 2020 Article reuse guidelines: sagepub.com/journals-permissions DOI: $10.1177 / 1557988320973826$ journals.sagepub.com/home/jmh

(S)SAGE

\author{
Alyssa K. Laserson, RN MN-NP(F)(C)' (D), John L. Oliffe, PhD, RN ${ }^{1,2}(\mathbb{D}$, \\ Jennifer Krist, DNP, NP(F)', and Mary T. Kelly, MA'
}

\begin{abstract}
The human papillomavirus (HPV) potentially affects every sexually active man in the United States and Canada. In 2017, the vaccine became publicly funded in Canada for males ages 9-26, and was integrated into school vaccination programs. In 2019, HPV vaccination was recommended as routine for all U.S.-based males and females ages 9 through 26 , and a shared decision for adults $>26$ years; however, since the approval of the vaccine in 2006 for females only, the age and dosing recommendations for males have followed a complicated and changing trajectory. Current adherence rates are low among college and university age males (18-26 years); therefore, understanding and addressing the barriers and facilitators for men's HPV vaccination is critically important. The purpose of the current scoping review is to provide a synthesis of recent literature pertaining to HPV in college and university age men, as a means to guiding health-care providers (HCPs). Drawing from 15 published articles, three thematic findings were inductively derived. Theme one, lack of awareness, was underpinned by men's knowledge deficits about their eligibility for, and the availability of HPV vaccines. Theme two, underestimating and embodying risk, included men's engagement in sexual activities while misinformed or denying the risk for contracting HPV. The third theme, strategies for increasing men's awareness, summarizes messaging strategies used to lobby young men to vaccinate. The review findings indicate gender-sensitive interventions targeting college-age men, including early, frequent, and consistent messaging on HPV are key.
\end{abstract}

\section{Keywords}

College men, special populations, sexual health, sexuality, public health, health-care issues

Received May 27, 2020; revised October 20, 2020; accepted October 23, 2020

More than $70 \%$ of sexually active individuals will contract human papillomavirus (HPV) at some point in their life (Canadian Public Health Agency, 2014). Other estimates of the lifetime probability of acquiring HPV are higher than $80 \%$ (Chesson et al., 2014). The first vaccine for HPV was introduced in the United States and Canada in 2006; at that time, it was recommended for females only, ages 9-26. In 2010, Health Canada expanded the recommendation for population-wide vaccination to include males of the same age range, although this strategy was not publicly funded until 2017. In the United States, 2010 permissive recommendations similarly extended HPV vaccination to males ages 9-26; in 2011, the vaccine received a routine recommendation for males ages 13-21, and a permissive recommendation for males 22-26 years. In 2019, routine recommendations changed to include males (and females) 11-26 years and shared decision-making for adults above 26 years. The history of the guidelines is complex and consistently denied males equal access to the health benefits of the vaccine, reflecting a schism between science, policy, and economics (Daley et al., 2017). In Canada, universal health-care and school programs cover the costs of the vaccine. In the United States, due to a lack of basic health-care coverage

\footnotetext{
'School of Nursing, University of British Columbia, Vancouver, BC, Canada

${ }^{2}$ Department of Nursing, University of Melbourne, Melbourne, Victoria, Australia

\section{Corresponding Author:}

Alyssa K. Laserson, RN MN-NP(F)(C), School of Nursing, University of British Columbia, T20I-22II Wesbrook Mall, Vancouver, BC V6T 2B5, Canada.

Email: alyssalaserson@gmail.com
} 
for all citizens, shifting vaccine recommendations resulted in additional complicated insurance issues; although public programs such as the federal Vaccines for Children exist, debates over school vaccine programs continue at the state level amid a patchwork of private and public coverage (Centers for Disease Control [CDC], 2019a; Kaiser Family Foundation, 2018; National Conference on State Legislatures (NCSL), 2020).

Since the introduction of the vaccine for men, adherence has been staggeringly low (Bird et al., 2017). According to a 2017 systematic review, male HPV vaccine adherence was only $47 \%$, well below the $>85 \%$ target set by the Canadian government (Bird et al., 2017). Similarly, in the United States, approximately $44 \%$ of teenage boys (aged 13-17) had the vaccine (Kaiser Family Foundation, 2018), while among male college students, $53 \%$ indicated they had received the vaccine (American College Health Association, 2019). Other research suggests that men's HPV vaccine completion rates (comprising a series of three HPV vaccines over 6 months at that time) were only 14\% (Johnson et al., 2017).

HPV can cause benign and malignant anogenital disease as well as head and neck lesions (Palefsky et al., 2011). Most HPV infections in males (and females) are asymptomatic, but often present as genital warts. The two HPV strains most attributable to anogenital warts are HPV types 6 and $11(>90 \%)$ and $20 \%-50 \%$ of cases involve a combined infection with oncogenic HPV types (Palefsky et al., 2011). HPV types 16 and 18 are most commonly associated with cancers of the penis, anus, and oropharynx (Palefsky et al., 2011); oropharyngeal cancers comprise approximately $80 \%$ of HPV-associated cancers in men each year (CDC, 2019b). HPV is also believed to cause $90 \%$ of anal cancers, $60 \%$ of penile cancers, and is linked to $70 \%$ of oropharynx cancers (CDC, 2019c). Since the infections are typically asymptomatic until life-changing progression occurs, vaccination is an especially important protection and ideally prevention strategy. To advance cancer prevention, it is essential that HPV vaccination be adapted by men and women.

\section{HPV and the Impact of Vaccination for College- and University-Age Males}

Upwards of 44,000 HPV-associated cancers occur in the United States each year; 19,000 are diagnosed in men, most commonly oropharyngeal cancer (CDC, 2019a, b, c). In 2020, there remains no Food and Drug Administration (FDA)-approved test available to detect the virus's DNA in males. Males are unlikely to know if they are infected with HPV, unless they have been diagnosed with anogenital warts, HPV-associated intraepithelial neoplasia, or HPV-related carcinoma (Workowski
\& Bolan, 2015). Males have a high potential for developing serious life-changing disease as well as unknowingly passing the virus on to their partners. Modeling predicting impacts of the vaccination on HPV type 16 infections and cervical cancer predicted that vaccination of $80 \%$ of 12-year-old girls would reduce HPV type 16 prevalence by $60 \%-100 \%$ in vaccinated, and $7 \%-31 \%$ in unvaccinated females; whereas, if $80 \%$ boys were also vaccinated, reductions would be $74 \%-100 \%$ in vaccinated and $86 \%-96 \%$ in unvaccinated females (Regan et al., 2007). A population study of HPV-associated cancers concluded that the HPV16/18 vaccine could prevent $60 \%$ of oropharyngeal cancers (Saraiya et al., 2015), which are most common in males. In June 2020, the FDA approved Gardasil 9 for the prevention of oropharyngeal cancer in males and females, ages 9-45; however, additional clinical trials required to demonstrate benefit are needed (F.D.A., 2020). With such potential reductions, the public health benefits of increasing HPV vaccine adherence among males are evident, and by extension, the avenues for achieving widespread uptake of the vaccine are critically important.

In the unique context of HPV vaccines, it is fair to say that men have been a latent target of messages to encourage vaccination (Pérez et al., 2015). To some extent, the afterthought of targeting men has revealed gender-blind public health practices that perhaps inadvertently led to lobbying women to take responsibility for HPV vaccination - and by extension prevention of HPV. Because the HPV vaccine was initially approved and introduced for females only, there may be lingering confusion among the public and health-care providers (HCPs) about its value for males. In tandem, the absence of tailored messaging for men has played to stereotypes that men do not engage self-health, a dominant discourse that can also mislead HCPs about the need to expect yields from actively promoting HPV vaccination in young men (Salgado et al., 2019).

\section{Purpose for Current Scoping Review}

With HPV vaccination adherence rates unacceptably low among college- and university-age males (18-26 years), the purpose of this scoping review was to provide a synthesis of recent relevant literature on young males and HPV vaccine as a means to guiding HCPs. Because the vaccination for males was not approved by the FDA and recommended by the National Advisory Committee on Immunizations (NACI) until 2012, and the guidelines for males did not extend through to age 26 until 2019 (Howard, 2019), the literature regarding how best to support and implement vaccine uptake within this population, is at best, emergent (Ratanasiripong, 2015; Salvadori, 2018). The vaccine is offered to males and females 
starting in grade six (ages 11-12) in the United States and Canada as part of routine vaccine schedule; however, there is no regularly scheduled follow-up once they finish high school and begin post-secondary education. In addition, there are inconsistencies across the U.S. states in regard to school vaccine policies (National Conference on State Legislatures (NCSL), 2020). After age 18, many young men navigate their health independent of family, making decisions on their own. Their estrangement from health-care services has been well documented, especially in the context of sexual health and sexually transmitted infection (STI) screening (Salgado et al., 2019). Therefore, an important first step in creating an effective intervention to increase HPV adherence is the exploration of college- and university-age men's practices, beliefs, and decision-making processes regarding vaccinations and health prevention. Understanding males' (18-26 years) perceptions of HPV risk and screening is critical to tailor clinical guidelines and raise awareness among men and HCPs about the benefits of HPV vaccination (Fontenot et al., 2014).

\section{Methods}

Scoping reviews are useful for producing knowledge syntheses about poorly understood health issues (Colquhoun et al., 2014; Peterson et al., 2017). Differentiating from systematic reviews and meta-analyses, the current scoping review includes diverse literature to point to emergent empirical insights, knowledge gaps, and potential next steps to advance male HPV vaccinations (Arksey \& O'Malley, 2005). In line with scoping review methods, the literature was not formally evaluated or weighted in terms of individual studies. Rather, empirical studies were reviewed as a means to understand and contribute recommendations for future research, education, practice, and policy (Peterson et al., 2017). Guided by Arksey \& O'Malley (2005), five key stages were followed in the current scope: (1) identifying the research question, (2) identifying relevant studies, (3) relevant study selection, (4) charting the data, and (5) collating, summarizing, and reporting the results.

Guided by the research question "What are the facilitators and barriers to HPV vaccination adherence among undergraduate U.S. and Canadian based college and university age males (18-26 years)?" Google Scholar, MEDLINE, and CINAHL databases were searched using the following keywords: papillomavirus vaccine, human papillomavirus vaccine, HPV vaccine, college student(s), university student(s), undergrad, men, male(s), young adult(s), and student(s), male HPV related cancer. The search terms were applied in a variety of combinations using Boolean operators and MeSH subject headings. Inclusion criteria included qualitative and quantitative research articles published between 2015 and 2019 inclusive, written in English, and focused on male populations or samples between the ages of 18 and 26 years.

In total, the searches returned 675 articles. Titles and abstracts of each article were read and appraised for their relevance to the topic and research question. Articles reporting female or female and male aggregate results, with their sample residing outside the United States and Canada, and the age range of 18-26 years old were excluded from the search results. Duplicate articles were also removed, resulting in 15 articles that met the inclusion criteria.

Each of the 15 articles were analyzed by first reading the article in full. To assist in organizing and classifying principal aspects of each article, a matrix table was developed by extracting the following elements: year of publication, country of origin, study purpose, design/ methodology, population and sample size, and important findings. The matrix provided a scaffold from which to inductively derive the prevailing themes along with key concepts identified across the studies (Table 1).

In categorizing the main findings of each study, three key themes were identified and further analyses and delineation of each were developed in the writing up of the results. Theme one, lack of awareness, was underpinned by men's knowledge deficits about their eligibility for, and the availability of HPV vaccines. Theme two, underestimating and embodying risk, included men's engagement in sexual activities while uninformed or denying the risk for contracting HPV. The third theme, strategies for increasing men's awareness, summarizes health messaging strategies used to lobby young men to vaccinate.

\section{Results}

The 15 North American-based studies included in the current scoping review consisted of the following methodologies: seven (46\%) quantitative, four (27\%) qualitative, and four (27\%) mixed-methods. Thirteen (86\%) studies were conducted in the United States and two $(13 \%)$ in Canada.

\section{Lack of Awareness About HPV}

Lack of awareness regarding HPV and the HPV vaccine was a consistent theme in over half the studies $(n=9$; $60 \%$ ). When asked about HPV and the vaccine, the results consistently revealed significant knowledge deficits wherein many men did not know the disease or vaccine existed (Catalano et al., 2017; Fontenot et al., 2014; Fuller \& Hinyard, 2017; Grace-Leitch \& Shneyderman, 2016; Hunter \& Weinstein, 2016; Pitts et al., 2017; Priest et al., 2015; Ratanasiripong, 2015; Tatar et al., 2017). 


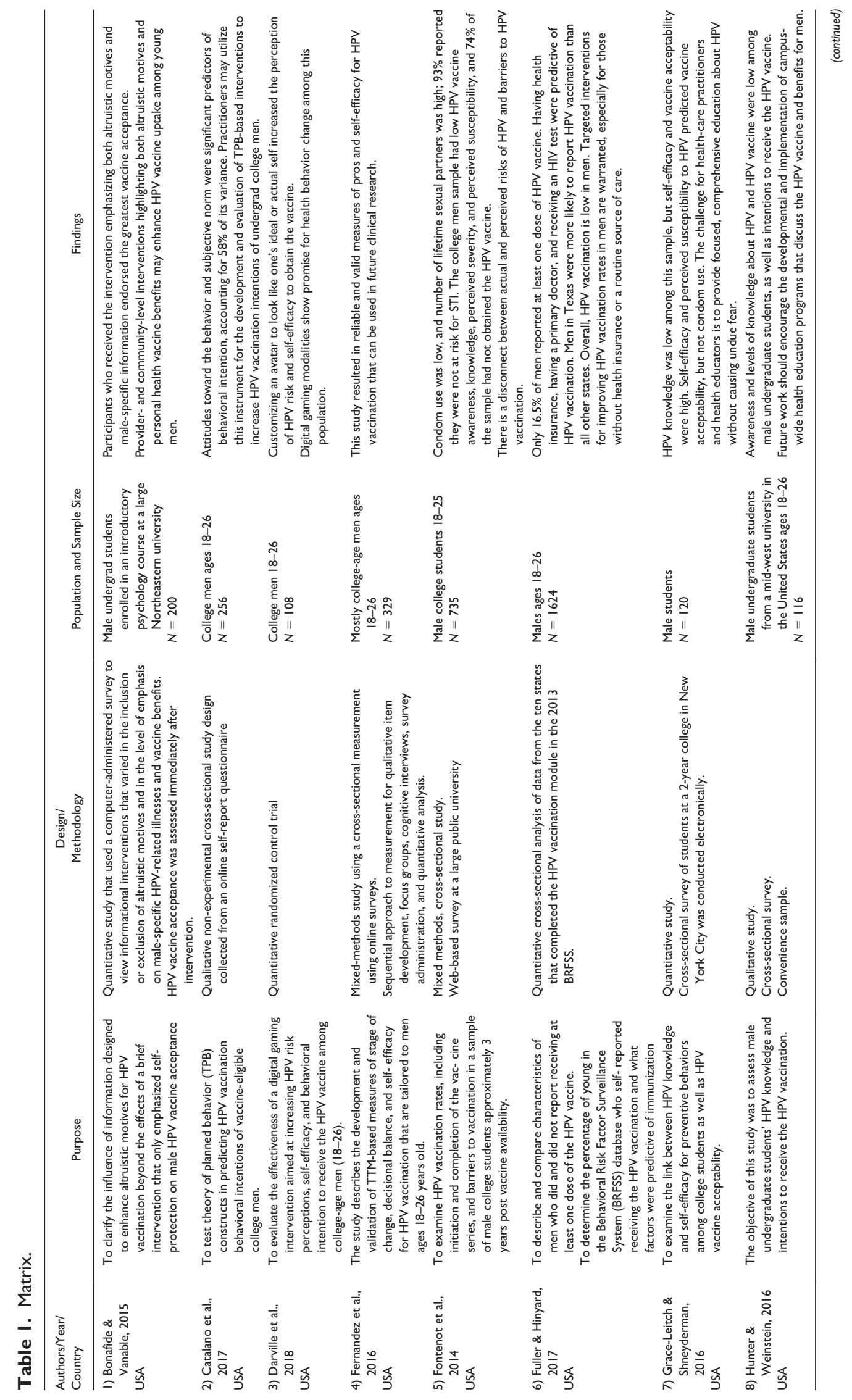




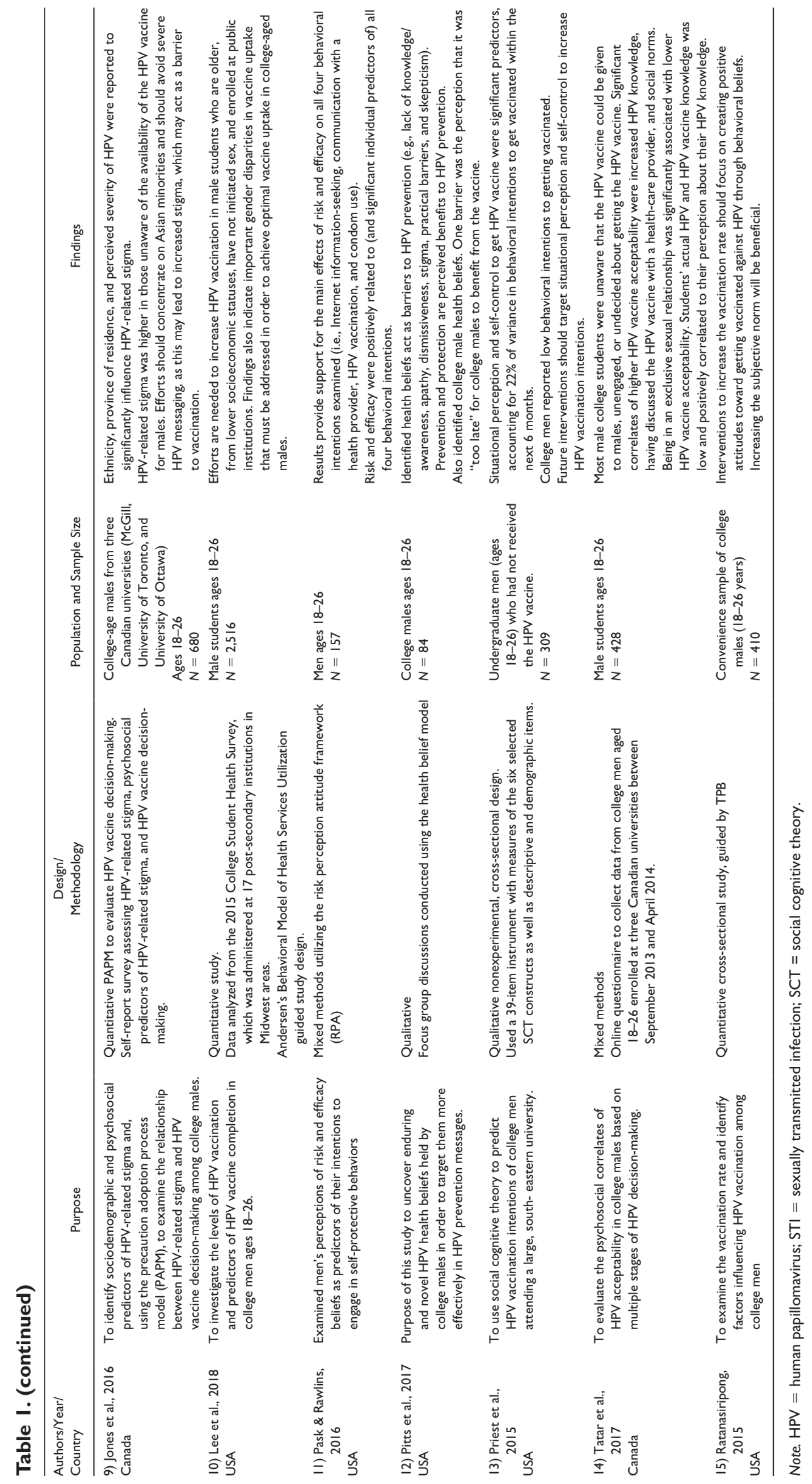


This lack of awareness and knowledge was the most commonly reported contributing factor for low vaccine rates and high levels of HPV-related illness (Catalano et al., 2017; Fontenot et al., 2014; Hunter \& Weinstein, 2016; Jones et al., 2016; Pitts et al., 2017; Priest et al., 2015; Ratanasiripong, 2015; Tatar et al., 2017). For example, Hunter and Weinstein (2016) noted that of those men surveyed, 35\% $(n=40)$ indicated that they had never heard of HPV and $51 \%(n=59)$ indicated they were unaware of the HPV vaccine before taking the survey. Among participants, $80 \%(n=93)$ also indicated that they did not know the HPV vaccine was available for men (Hunter \& Weinstein, 2016). Similarly, another study reported that $31.3 \%(n=80)$ of the sample had never heard of HPV, and $45.3 \%(n=116)$ were unaware of the vaccine, even though $86.3 \%(n=221)$ reported having previously engaged in oral, vaginal, and/or anal sex (Catalano et al., 2017). Vaccine-related decision-making was correlated to awareness and knowledge levels about the HPV infection and negative health outcomes, as well as the existence of the vaccine and its availability for men (Pitts et al., 2017; Priest et al., 2015; Tatar et al., 2017).

Several studies indicated that although some participants had heard about HPV and the vaccine, when asked specific questions regarding the disease and prevention, significant knowledge deficits were evident (Pitts et al., 2017; Priest et al., 2015). Similar results were reported in a Canadian study wherein only $21.5 \%(n=92)$ of respondents indicated being aware that most sexually active people get HPV at some point in their lives; $29.9 \%(n=$ 128) knew that the HPV vaccine offered protection against genital warts, and $32.7 \%(n=140)$ were knowledgeable about the number of doses required to achieve adequate protection (Tatar et al., 2017). These statistically significant results indicated only one-fifth of the participants $(19.46 \%, n=83)$ accurately categorized themselves as having sufficient HPV knowledge (Tatar et al., 2017).

Because HPV knowledge and awareness are so limited in males, their understanding of the consequences of contracting HPV is also low (Fontenot et al., 2014). In one study, only $56 \%$ of participants $(n=123)$ were able to correctly identify issues related to HPV, indicating that nearly half were unaware of the symptoms (or lack of symptoms), consequences, and/or risks associated with an HPV infection (Grace-Leitch \& Shneyderman, 2016). Several studies reported that little to no understanding about HPV could lead to negative health complications for men, an outcome directly associated with lack of STI knowledge (Fontenot et al., 2014; Hunter \& Weinstein, 2016; Priest et al., 2015; Tatar et al., 2017). In other research that surveyed participants on their HPV knowledge, only $45 \%(n=139)$ were aware that HPV can cause cancer, and only $62 \%(n=192)$ of participants were aware that HPV could cause genital warts (Priest et al., 2015). Similar findings were reported by Ratanasiripong (2015); less than or approximately half the participants $(n=189)$ knew that HPV can cause anal cancer, and/or genital warts, and be transmitted via skin-to-skin contact. Widespread misperceptions of HPV including minimizing the severity and/or susceptibility in males have led to a largely dismissive attitude to vaccination (Fontenot et al., 2014).

\section{Underestimating and Embodying Risk}

Present in over half the articles ( $n=9 ; 60 \%)$, underestimating risk was characterized by men's lack of perceived risk and susceptibility for contracting HPV. A common misconception among college and university age men was the belief they were not at risk for contracting HPV. Many believed HPV affected females only (Fontenot et al., 2014; Tatar et al., 2017). For example, the Tatar (2017) study reported that $74.8 \%(n=320)$ of respondents believed men could not contract HPV (Tatar et al., 2017). Condom use, knowing their partners' sexual history, and/or believing they had already been exposed to HPV in the past were offered as risk-reducing and -protective factors by men (Fontenot et al., 2014). Among Canadian men, $81.3 \%(n=347)$ were aware that condom use reduced HPV transmission (Tatar et al., 2017). Similar results were identified in a U.S.-based study where $84 \%$ $(n=159)$ participants were aware that condoms provided partial protection against HPV (Ratanasiripong, 2015). The misconceptions that condoms provided full protection against HPV lead to underestimation of risk and lower vaccine rates (Ratanasiripong, 2015; Tatar et al., 2017). Among men with basic knowledge of HPV risk factors (i.e., multiple sexual partners, not using condoms), $93 \%(n=683)$ self-reported they were not at risk for contracting an STI, illustrating a disconnect between actual and perceived risk (Fontenot et al., 2014). Apathy about, and dismissiveness toward, HPV and its health implications were a commonly reported finding (Fontenot et al., 2014; Pitts et al., 2017; Ratanasiripong, 2015). One study indicated that $68.9 \%(n=295)$ respondents believed that HPV was a very rare disease (Tatar et al., 2017). Male preconceptions of invincibility were also a factor in the lack of preventative practices (Pitts et al., 2017).

Young adult males (18-26 years) expressed the perspective that it was too late to get the vaccination given their active sexual history, and believed they no longer qualified to receive the vaccination, putting them at increased risk (Pitts et al., 2017). Another barrier that men reported was the stigma associated with STIs and the perception that vaccination might be misconstrued as treatment for HPV (Pitts et al., 2017). In other words, 
stigma was a more potent influence than health risks for many young men. Risk was also minimized when contraction of HPV was perceived as less serious than other STIs (i.e., HIV; Fontenot et al., 2014; Pitts et al., 2017). The role of media was cited as influencing some men's alignment to risky sexual behaviors and promiscuity in tandem with the dismissal of the importance of HPV vaccine due to the information being presented and the lack of male inclusion (Bonafide \& Vanable, 2015; Perez et al., 2015).

\section{Strategies for Increasing Men's Awareness}

The pivotal role that HCPs can play in engaging and encouraging HPV vaccine adherence was a common finding in $53 \%(n=8)$ studies (Bonafide \& Vanable, 2015; Fontenot et al., 2014; Fuller \& Hinyard, 2017; Hunter \& Weinstein, 2016; Lee et al., 2018; Priest et al., 2015; Ratanasiripong, 2015; Tatar et al., 2017). In this regard, HCPs played an integral role in both creating awareness and education opportunities about HPV, and administration of the HPV immunization. Even a simple conversation with an HCP was reported to increase HPV vaccine adherence in university- and college-age males (Fuller \& Hinyard, 2017; Hunter \& Weinstein, 2016; Tatar et al., 2017). In one study, $43 \%(n=24)$ students who initiated discussion with an HCP received a positive recommendation to vaccinate (Tatar et al., 2017). As male adolescents become adults, however, the opportunity to have these health-promoting conversations diminished because routine health-care visits were low among young men. Healthy young adult males were lost to routine medical care and check-ups, and were therefore unlikely to engage these types of conversations with their HCP (Fontenot et al., 2014).

A major barrier to vaccine uptake reported in several studies was simply that college-age men had never been offered the vaccine by their HCPs (Bonafide \& Vanable, 2015; Fontenot et al., 2014; Fuller \& Hinyard, 2017; Hunter \& Weinstein, 2016). Among the 116 participants in one study, only $6 \%(n=7)$ indicated they had received the HPV vaccine, all of whom stated that they were informed about the vaccine by an HCP (Hunter \& Weinstein, 2016). Similar findings were reported in another study in which $60.9 \%(n=989)$ participants indicated the most common reason for not receiving the vaccine was that their HCP had not recommended it (Fuller \& Hinyard, 2017). Likewise, a Canadian study indicated that $81 \%(n=347)$ male participants (ages 18-26) had visited an HCP in the past year; however, $87 \%(n=373)$ suggested that their HCP had never discussed the HPV vaccine with them (Tatar et al., 2017).

Lack of access to a regular HCP was listed as another direct barrier to receiving education and ongoing reminders about health preventative practices including the HPV vaccine. In a U.S. study, $51 \%(n=684)$ participants unvaccinated against HPV reported that they did not have a primary HCP; whereas $72 \%(n=203)$ of the vaccinated participants reported that they did access an HCP (Fuller \& Hinyard, 2017). Men with a regular HCP were almost two and half times more likely to have received an HPV vaccine compared to those without an HCP (Fuller \& Hinyard, 2017). Most of the males who received the vaccine $(75 \%, n=207)$ also reported having access to an HCP (Fuller \& Hinyard, 2017). With collegeand university-age men being the subpopulation most often without an HCP, providers should use every opportunity to enhance and educate young men on appropriate self-care practices (Bonafide \& Vanable, 2015). Despite this important strategy in HPV vaccine adherence, studies with HCPs have revealed that HCPs are more likely to recommend the vaccine to female patients than male patients (Lee et al., 2018), contributing to a significant gender bias regarding HPV.

Conversations about preventative health behaviors at an early age fostered normative beliefs about prevention regarding safe sexual health practices. For example, a U.S. study suggested that to enhance positive attitudes toward HPV vaccination, interventions should focus on behavioral beliefs of young males and this could be achieved by frequent and early messaging both from parents and from sexual health education delivered by HCPs (Ratanasiripong, 2015). Having a parent who held a graduate degree was significantly associated with the higher odds of male child HPV vaccine completion (Lee et al., 2018). In two studies, the age of the male was the only significant demographic characteristic associated with HPV vaccination (Fontenot et al., 2014; Ratanasiripong, 2015). On a continuous range from 18 to 25 years, there were $24 \%$ lower odds of having obtained the HPV vaccine for every year these young men grew older (Fontenot et al., 2014). These findings indicate the need to increase HPV knowledge among parents and introduce health education at earlier ages, and ideally these prevention practices will carry forward into adulthood.

Several studies concluded that the communication of altruism in conjunction with HPV information could improve vaccination rates among males. When HPV knowledge included the benefits to men's partners and broader health benefits at the global level, vaccine recommendations were more likely to be taken up (Bonafide \& Vanable, 2015). In a U.S. study, increased acceptance occurred when participants were given information about the HPV vaccine for males plus altruistic motivations compared to participants who received the male-focused HPV information only (Bonafide \& Vanable, 2015). Altruistic motivation was identified in another study in which men's recognition of the broader social benefits of 
HPV prevention for others was highlighted as a motivating factor to vaccinate (Pitts et al., 2017). A novel strategy was evaluated in a digital gaming randomized control trial (RCT) designed to improve vaccine outcomes among young male college participants; results demonstrated how the use of customized avatars increased men's risk perception, self-efficacy, and behavior intention to receive the vaccine (Darville et al., 2018).

\section{Discussion}

This scoping review was conducted to better understand the facilitators and barriers to HPV vaccination adherence among U.S. and Canadian undergraduate collegeand university-age males (18-26 years), and to recommend how HCPs can use this knowledge to guide practice, influence change, and establish improved men's health promotion strategies. The chief areas identified in this review were men's lack of awareness, their underestimation of risk, and the need for male-focused early intervention. However, given the complex history and series of changes in vaccine policy recommendations for males, it is not surprising that the findings confirmed that men's understanding of risk and overall HPV knowledge was limited.

HCPs can focus on these areas to increase HPV vaccination rates among men. Perhaps the most important finding in this review is the influence that HCPs carry related to vaccine adherence. HCPs are well-positioned to educate and advocate for the importance of the HPV vaccine (Bonafide \& Vanable, 2015; Fontenot et al., 2014; Fuller \& Hinyard, 2017; Hunter \& Weinstein, 2016; Lee et al., 2018; Priest et al., 2015; Ratanasiripong, 2015; Tatar et al., 2017). There is consensus beyond this review that vaccine adherence increases with HCP recommendations (Alexander et al., 2015; Allison et al., 2016; Gerend et al., 2016). However, it is challenging to define practice guidelines regarding the specific current findings due to the wide-ranging variables influencing diverse patient populations.

Standardized HPV education and information practices for HCPs are in progress. However, HCPs should concentrate on repeated dissemination of information about HPV and the vaccine at every relevant opportunity. The most important reminder for HCPs when approaching this topic with patients and families is that the spectrum of readiness with adolescent sexual activity varies greatly and information must be provided in a familycentered approach, with appropriate and careful consideration for the specific individuals (Money et al., 2007). Professional organizations continue to reinforce that vaccination against HPV is the best primary prevention method, and that screening for asymptomatic HPV infections is not possible because there is no validated test for men (Money et al., 2007; Saslow et al., 2016). Guidelines from the American Cancer Association reinforce the notion that opportunities should be presented for young men in college to be vaccinated, and that young adults should not require parental consent to receive the HPV vaccine.

Despite the influential role of HCPs in educating males about the benefits of HPV vaccination, HCPs often forfeit or miss those opportunities during consults (Cummings et al., 2015). Daley et al. (2017) recounted the historical "feminization of HPV" and the complicated guidelines, dosing schedules, and conflicting information from the FDA and Advisory Committee on Immunization Practices (ACIP) for over a decade. That complex history may have contributed to vaccine gender biases among HCPs as well. Complicating the role of the HCPs in vaccine compliance is the fact that increasingly many Americans and Canadians are without a primary HCP. In Canada during 2016, $15.8 \%$ of citizens (approximately 4.8 million people) over 12 years of age were without a primary HCP. Significantly more males $(19.9 \%)$ compared to females $(11.9 \%)$ were without a primary $\mathrm{HCP}$, and males 18-34 years old were more likely than any other age group to be without a regular HCP (Statistics Canada, 2016). Similar results have been noted in the United States; $28 \%$ of men compared to $18 \%$ of women over the age of 18 were without a regular HCP. Lack of access and connectedness to HCPs for a significant portion of the population implies that there is an increased likelihood that HPV health messaging cannot be disseminated to college-age males.

Media plays an important role as a source of public information about HPV. Because the HPV vaccine was initially framed as a female responsibility, publicly funded for women only with advertising explicitly targeting females, public health campaigns are playing catch-up to recalibrate messaging to include males. Two significant findings from this review could be incorporated into health promotions messaging and HCP discussions. First, changing attitudes toward HPV vaccination and gender norms can significantly predict intentions to vaccinate (Ratanasiripong, 2015). If education about HPV and sexual health starts at a young age, positive values and practices toward sexual health and STI prevention can result in increased vaccine adherence. Second, findings suggested that stressing both the male-specific health benefits as well as altruistic motives such as protecting others by vaccinating can improve male receptivity to vaccination. Advertising campaigns, public health messaging, school vaccination programs, and HCP consults may achieve greater impact on male vaccine adherence by pairing these ideas (Bonafide \& Vanable, 2015). The role of altruism in young men's health has been confirmed in other research. A Canadian 
study investigating masculine norms in relation to health care reported that young men (ages 15-29) consider putting the welfare of others ahead of their own to be a deeply valued positive masculine behavior (Oliffe et al., 2019). The use of video gaming to creatively deliver messaging about HPV to young college-age men also plays to normative masculinities and values such as competitiveness, promoting behavior intent to receive the vaccine (Darville et al., 2018).

The most common misconceptions were perceptions that young men believed it was too late to be vaccinated and that HPV only affects women. This misinformation illustrated the lack of understanding about perceived timing, risk, and the severity of HPV-related illness (Hunter \& Weinstein, 2016; Pitts et al., 2017; Priest et al., 2015; Ratanasiripong, 2015). The lack of perceived risk (Fontenot et al., 2014; Hunter \& Weinstein, 2016; Pask \& Rawlins, 2016) was directly related to both lack of awareness and the well-documented absence of sexual health-seeking behaviors typically seen in young males (Salgado et al., 2019). Lack of HPV knowledge combined with high-risk sexual behaviors in late adolescence and early adulthood highlight the need for health promotion strategies to prevent lifechanging illnesses (Crocker et al., 2019). Communitybased health promotion programs need to be tailored specifically to young men's needs and counter normative beliefs and misperceptions about risk.

In order to enhance positive attitudes toward HPV vaccine adherence, interventions altering behavioral and beliefs (e.g., having anogenital cancer is negative and getting the vaccine protects men from serious illness) can enhance and transform subjective norms (Ratanasiripong, 2015). HCPs can develop educational programs to increase patient education and awareness about HPV; evaluate current health center policies and procedures to assess for known barriers; and support an environment that promotes vaccination (Fontenot et al., 2014). Although educating males on the risks of HPV is vital, the focus of interventions should not be on the severity of HPV; heightened perceptions of HPV severity can lead to higher HPV-related stigma, which in turn may act as a barrier to vaccination (Jones et al., 2016). Stigma is a more influential barrier to vaccine adherence when participants are in early stages of HPV vaccine decisionmaking, namely "unaware" (Jones et al., 2016). Health promotion campaigns should focus on educational facts of HPV without fueling stigmas or anxieties surrounding HPV. Information presented to college-age men should emphasize that although the vaccine is most effective with the series completed prior to sexual debut, the vaccine continues to be beneficial as a preventative measure even after a person has engaged in sexual activities (Pitts et al., 2017).
Due to extended approval of the vaccine for men, there needs to be a significant shift in how information is presented to college- and university-age men (18-26; Catalano et al., 2017; Fontenot et al., 2014; Hunter \& Weinstein, 2016; Pitts et al., 2017; Priest et al., 2015). Masculine values as they pertain to male health care are highly relevant for a disease and treatment that have previously been highly feminized. This pivot in public health policy may explain why the current review reported that significant barriers to vaccination were male perceptions of the HPV vaccination as feminine, and therefore not applicable and perhaps stigmatizing.

Gendered health behaviors are learned and may be socialized in men from an early age, reinforced during adolescence, and amplified in early adulthood (Crocker et al., 2019). Studies have demonstrated that aligning to traditional masculine norms predicts men's low rates of engagement in preventative health behaviors, such as routine medical exams, self-care practices, and psychological help (Salgado et al., 2019). Men who do disclose engaging in sexual health promoting practices risk being characterized as "feminine" or "weak" (Salgado et al., 2019). Often, male-biased stereotypes may alter the HCP's perception of how men receive information, and they may withhold discussions about HPV that otherwise may have been well received. For example, female physicians have been reported to provide patients with more preventive and counseling services than male physicians (Bertakis, 2009). Dominant expressions of traditional masculinity, such as competitiveness, risk-taking, self-reliance, stoicism, and being perceived as heterosexual, are still widespread, and these norms encourage men to restrict self-protective health behaviors (Salgado et al., 2019). To counter masculine stereotypes, health promotion programs should focus on young men's susceptibility to HPV and work to shift normative behaviors around sexual health (Grace-Leitch \& Shneyderman, 2016). Health messaging should target men on college campuses, especially in light of findings indicating that increased knowledge and perception of HPV susceptibility improved vaccine acceptability among young men. Future research should aim to understand how messaging impacts vaccine acceptance and adherence, including among males who do not identify with "stereotypical" masculine norms (Bonafide \& Vanable, 2015; Lee et al., 2018).

\section{Limitations}

This review is intended to guide practice for HCPs; however, there are several key limitations. The major limitation in this set of articles is that all but one $(n=14)$ of the included studies (Fuller \& Hinyard, 2017) gathered participants directly from post-secondary institutions. This 
sampling method limits the findings in terms of understanding males who may not be attending conventional post-secondary institutions (i.e., college or university) and/or those from low socioeconomic backgrounds considered at high risk for contracting HPV, but who may not be afforded the same level of health promotion and prevention opportunities. The second limitation is that all the included studies were comprised exclusively of male samples. The absence of female perspectives creates a potentially segregated view of HPV, when in reality, HPV prevention needs to focus on genders equally, equitably, and relationally. Future work should focus on combined male and female strategies within college and university age ranges to decrease barriers. Such targeting is essential to creating a broader understanding and increasing the likelihood of a population effect on HPV vaccine adherence rates.

\section{Conclusion}

Because the HPV vaccine recommendations were repeatedly updated for males over the last 10 years, evidence is still emerging on how best to increase vaccine adherence. With rates of HPV-related illnesses on the rise, strategies to mitigate this growing health concern must be established quickly in order to decrease related illness and deaths. The focus must be on health promotion and prevention approaches geared specifically to males. Norming male HPV vaccinations is key, and the work of that clearly resides with men and HCPs, parents, partners, and public health in redressing the gendered history that estranged men in the first place.

\section{Acknowledgments}

To my unwavering supervisors, Dr. Oliffe and Dr. Krist-you both inspired me throughout this entire process. Thanks to the Men's Health Research Program and the UBC School of Nursing for their financial support in covering the open access fees for the current article. Thank you for helping make my words and writing change future practice. This is dedicated to my husband Jeffrey who is my light, my life, and the biggest pillar of support.

\section{Declaration of Conflicting Interests}

The author(s) declared no potential conflicts of interest with respect to the research, authorship, and/or publication of this article.

\section{Funding}

The author(s) received no financial support for the research, authorship, and/or publication of this article.

\section{ORCID iDs}

Alyssa K. Laserson (iD https://orcid.org/0000-0002-7498-8504

John L. Oliffe (ID https://orcid.org/0000-0001-9029-4003

\section{References}

Alexander, A. B., Best, C., Stupiansky, N., \& Zimet, G. D. (2015). A model of health care provider decision making about HPV vaccination in adolescent males. Vaccine. https://doi.org/10.1016/j.vaccine.2015.06.085

Allison, M. A., Hurley, L. P., Markowitz, L., Crane, L. A., Brtnikova, M., Beaty, B. L., Snow, M., Cory, J., Stokley, S., Roark, J., \& Kempe, A. (2016). Primary care physicians' perspectives about HPV vaccine. Pediatrics. https:// doi.org/10.1542/peds.2015-2488

American College Health Association. (2019). American College Health Association-National College Health Assessment II: Undergraduate Student Reference Group Data Report Spring 2019. American College Health Association.

Arksey, H., \& O’Malley, L. (2005). Scoping studies: Towards a methodological framework. International Journal of Social Research Methodology, 8(1), 19-32.

Bertakis, K. D. (2009). The influence of gender on the doctorpatient interaction. Patient Education and Counseling, 76(3), 356-360.

Bird, Y., Obidiya, O., Mahmood, R., Nwankwo, C., \& Moraros, J. (2017). Human papillomavirus vaccination uptake in canada: A systematic review and meta-analysis. International Journal of Preventive Medicine. https://doi.org/10.4103/ ijpvm.IJPVM_49_17

Bonafide, K. E., \& Vanable, P. A. (2015). Male human papillomavirus vaccine acceptance is enhanced by a brief intervention that emphasizes both male-specific vaccine benefits and altruistic motives. Sexually Transmitted Diseases. https://doi.org/10.1097/OLQ.0000000000000226

Canadian Public Health Agency. (2014). Human Papillomavirus (HPV). https://www.canada.ca/en/public-health/services/ infectious-diseases/sexual-health-sexually-transmittedinfections/human-papillomavirus-hpv.html

Catalano, H. P., Knowlden, A. P., Birch, D. A., Leeper, J. D., Paschal, A. M., \& Usdan, S. L. (2017). Using the theory of planned behavior to predict HPV vaccination intentions of college men. Journal of American College Health. https:// doi.org/10.1080/07448481.2016.1269771

CDC. (2019b). Cancers associated with human papillomavirus United States - 2012-2016. https://www.cdc.gov/ cancer/uscs/pdf/USCS-DataBrief-No10-August2019-h. pdf

CDC. (2019c). HPV-associated cancer statistics. https://www. cdc.gov/cancer/hpv/statistics/

Centers for Disease Control and Prevention (CDC). (2019a, August). HPV vaccine schdeule and dosing. https://www. cdc.gov/hpv/hcp/schedules-recommendations.html

Chesson, H. W., Dunne, E. F., Hariri, S., \& Markowitz, L. E. (2014). The estimated lifetime probability of acquiring human papillomavirus in the United States. Sexually Transmitted Diseases, 41(11), 660-664.

Colquhoun, H. L., Levac, D., O’Brien, K. K., Straus, S., Tricco, A. C., Perrier, L., Kastner, M., \& Moher, D. (2014). Scoping reviews: Time for clarity in definition, methods, and reporting. Journal of Clinical Epidemiology, 67(12), 1291-1294.

Crocker, B. C. S., Pit, S. W., Hansen, V., John-Leader, F., \& Wright, M. L. (2019). A positive approach to adolescent 
sexual health promotion: A qualitative evaluation of key stakeholder perceptions of the Australian Positive Adolescent Sexual Health (PASH) Conference. BMC Public Health. https://doi.org/10.1186/s12889-019-6993-9 Cummings, T., Kasting, M. L., Rosenberger, J. G., Rosenthal, S. L., Zimet, G. D., \& Stupiansky, N. W. (2015). Catching up or missing out? Human papillomavirus vaccine acceptability among 18-to 26-year-old men who have sex with men in a US national sample. Sexually Transmitted Diseases, 42(11), 601-606.

Daley, E. M., Vamos, C. A., Thompson, E. L., Zimet, G. D., Rosberger, Z., Merrell, L., \& Kline, N. S. (2017). The feminization of HPV: How science, politics, economics and gender norms shaped US HPV vaccine implementation. Papillomavirus Research, 3, 142-148.

Darville, G., Anderson-Lewis, C., Stellefson, M., Lee, Y. H., MacInnes, J., Pigg Jr, R. M., Gilbert, J. E., \& Thomas, S. (2018). Customization of avatars in a HPV digital gaming intervention for college-age males: An experimental study. Simulation \& Gaming, 49(5), 515-537.

F.D.A. (2020). Gardisil 9. https://www.fda.gov/vaccines-bloodbiologics/vaccines/gardasil-9

Fernandez, A. C., Amoyal, N. R., Paiva, A. L., \& Prochaska, J. O. (2016). Motivation for HPV vaccination among young adult men: Validation of TTM decisional balance and self-efficacy constructs. American Journal of Health Promotion, 30(3), 163-171.

Fontenot, H. B., Fantasia, H. C., Charyk, A., \& Sutherland, M. A. (2014). Human papillomavirus (HPV) risk factors, vaccination patterns, and vaccine perceptions among a sample of male college students. Journal of American College Health. https://doi.org/10.1080/07448481.2013.872649

Fuller, K. M., \& Hinyard, L. (2017). Factors associated with HPV vaccination in young males. Journal of Community Health. https://doi.org/10.1007/s10900-017-0361-4

Gerend, M. A., Shepherd, M. A., Lustria, M. L. A., \& Shepherd, J. E. (2016). Predictors of provider recommendation for HPV vaccine among young adult men and women: Findings from a cross-sectional survey. Sexually Transmitted Infections. https://doi.org/10.1136/sextrans-2015-052088

Grace-Leitch, L., \& Shneyderman, Y. (2016). Using the health belief model to examine the link between HPV knowledge and self-efficacy for preventive behaviors of male students at a two-year college in New York city. Behavioral Medicine. https://doi.org/10.1080/08964289.2015.1121131

Howard, J. (2019). CNN. CDC panel recommends HPV vaccine for men through age 26 and for some older adults. https://www.cnn.com/2019/06/26/health/hpv-vaccine-agerecommendations-acip-bn/index.html

Hunter, T., \& Weinstein, M. (2016). Beliefs and knowledge about the human papillomavirus vaccine among undergraduate men. Health Education Journal. https://doi. org/10.1177/0017896915572705

Johnson, K. L., Lin, M. Y., Cabral, H., Kazis, L. E., \& Katz, I. T. (2017). Variation in human papillomavirus vaccine uptake and acceptability between female and male adolescents and their caregivers. Journal of Community Health. https://doi.org/10.1007/s10900-016-0284-5
Jones, G., Perez, S., Huta, V., Rosberger, Z., \& Lebel, S. (2016). The role of human papillomavirus (HPV)-related stigma on HPV vaccine decision-making among college males. Journal of American College Health. https://doi.org/10.10 80/07448481.2016.1192542

Kaiser Family Foundation. (2018). The HPV vaccine: Access and use in the USA. Retrieved from https://www.kff.org/ womens-health-policy/fact-sheet/the-hpv-vaccine-accessand-use-in-the-u-s/

Lee, H. Y., Lust, K., Vang, S., \& Desai, J. (2018). Male undergraduates' HPV vaccination behavior: Implications for achieving HPV-associated cancer equity. Journal of Community Health. https://doi.org/10.1007/s10900-0180482-4

Money, D. M., Roy, M., Scrivener, J., Allen, L., Brewer, M., Bryson, P., Evans, G., Frappier, J.-Y., Jamieson, M. A., Lynde, C., Murphy, K. J., Provencher, D. M., Shier, M., Steben, M., Lerch, R., Lalonde, A., \& Senikas, V. (2007). Canadian consensus guidelines on human papillomavirus. Journal of Obstetrics and Gynaecology Canada. https:// doi.org/10.1016/S1701-2163(16)32573-7

National Conference on State Legislatures (NCSL). (2020, May). HPV vaccine: State legislation and regulation. https://www.ncsl.org/research/health/hpv-vaccine-statelegislation-and-statutes.aspx

Oliffe, J. L., Rice, S., Kelly, M. T., Ogrodniczuk, J. S., Broom, A., Robertson, S., \& Black, N. (2019). A mixed methods study of the health-related masculine values among young Canadian men. Psychology of Men \& Masculinity, 20(3), 310-323.

Palefsky, J. M., Giuliano, A. R., Goldstone, S., Moreira, E. D., Aranda, C., Jessen, H., Hillman, R., Ferris, D., Coutlee, F., Stoler, M. H., Marshall, J. B., Radley, D., Vuocolo, S., Haupt, R. M., Guris, D., \& Garner, E. I. O. (2011). HPV vaccine against anal HPV infection and anal intraepithelial neoplasia. New England Journal of Medicine. https://doi. org/10.1056/NEJMoa1010971

Pask, E. B., \& Rawlins, S. T. (2016). Men's intentions to engage in behaviors to protect against Human Papillomavirus (HPV): Testing the risk perception attitude framework. Health Communication. https://doi.org/10.1080/10410236 .2014 .940670

Perez, S., Shapiro, G. K., Brown, C. A., Dube, E., Ogilvie, G., \& Rosberger, Z. (2015). "I didn't even know boys could get the vaccine": Parents' reasons for human papillomavirus (HPV) vaccination decision making for their sons. PsychoOncology. https://doi.org/10.1002/pon.3894

Peterson, J., Pearce, P. F., Ferguson, L. A., \& Langford, C. A. (2017). Understanding scoping reviews: Definition, purpose, and process. Journal of the American Association of Nurse Practitioners, 29(1), 12-16.

Pitts, M. J., Stanley, S. J., \& Kim, S. (2017). College males' enduring and novel health beliefs about the HPV vaccine. Health Communication. https://doi.org/10.1080/10410236. 2016.1196421

Priest, H. M., Knowlden, A. P., \& Sharma, M. (2015). Social cognitive theory predictors of human papillomavirus vaccination intentions of college men at a southeastern university. 
International Quarterly of Community Health Education. https://doi.org/10.1177/0272684X15583289

Ratanasiripong, N. T. (2015). Factors related to Human Papillomavirus (HPV) vaccination in college men. Public Health Nursing. https://doi.org/10.1111/phn.12198

Regan, D. G., Philp, D. J., Hocking, J. S., \& Law, M. G. (2007). Modelling the population-level impact of vaccination on the transmission of human papillomavirus type 16 in Australia. Sexual Health, 4(3), 147-163.

Salgado, D. M., Knowlton, A. L., \& Johnson, B. L. (2019). Men's health-risk and protective behaviors: The effects of masculinity and masculine norms. Psychology of Men and Masculinity. https://doi.org/10.1037/men0000211

Salvadori, M. I. (2018). Human papillomavirus vaccine for children and adolescents. Paediatrics and Child Health (Canada). https://doi.org/10.1093/pch/pxx179

Saraiya, M., Unger, E. R., Thompson, T. D., Lynch, C. F., Hernandez, B. Y., Lyu, C. W., Steinau, M., Watson, M., Wilkinson, E. J., Hopenhayn, C., \& Copeland, G. (2015).
US assessment of HPV types in cancers: Implications for current and 9-valent HPV vaccines. JNCI: Journal of the National Cancer Institute, 107(6), 1-12.

Saslow, D., Andrews, K. S., Manassaram-Baptiste, D., Loomer, L., Lam, K. E., Fisher-Borne, M., Smith, R. A., \& Fontham, E. T. H. (2016). Human papillomavirus vaccination guideline update: American Cancer Society guideline endorsement. CA: A Cancer Journal for Clinicians. https://doi. org/10.3322/caac. 21355

Tatar, O., Perez, S., Naz, A., Shapiro, G. K., \& Rosberger, Z. (2017). Psychosocial correlates of HPV vaccine acceptability in college males: A cross-sectional exploratory study. Papillomavirus Research. https://doi.org/10.1016/j. pvr.2017.11.001

Workowski, K. A., \& Bolan, G. A. (2015). Sexually transmitted diseases treatment guidelines, 2015. MMWR. Recommendations and reports: Morbidity and mortality weekly report. Recommendations and reports, 64(RR03), 1. 


\section{University Library}

\section{- M M I E E R VA A gateway to Melbourne's research publications}

Minerva Access is the Institutional Repository of The University of Melbourne

\section{Author/s:}

Laserson, AK;Oliffe, JL;Krist, J;Kelly, MT

Title:

HPV Vaccine and College-Age Men: A Scoping Review

\section{Date:}

2020-11-01

Citation:

Laserson, A. K., Oliffe, J. L., Krist, J. \& Kelly, M. T. (2020). HPV Vaccine and CollegeAge Men: A Scoping Review. AMERICAN JOURNAL OF MENS HEALTH, 14 (6), https:// doi.org/10.1177/1557988320973826.

Persistent Link:

http://hdl.handle.net/11343/281192

License:

CC BY-NC 